\title{
MODELLING AND VIBRATIONAL ANALYSIS OF FRONT LOADED WASHING MACHINE
}

\author{
Vishal S Patil ${ }^{1}$, A V Patil ${ }^{2}$ \\ ${ }^{I}$ ME Student, Department of Mechanical, SSGB COE, Maharashtra, India \\ ${ }^{2}$ Associate Professor and HOD, Department of Mechanical, SSGB COE, Maharashtra, India
}

\begin{abstract}
Recent environmental awareness requires the improvement of washing efficiency. Talking about efficiency, the first we needs to concentrate on optimization. Basically we need to concentrate on the parameters like supply voltages, mechanical parameters and etc. Many of the above parameters can be approximately optimised by solving the problem of Vibration. In a washing machine, vibration of the outer tub is responsible for vibration of the frame and the force then transfers from the supports to the floor. Although springs and dampers also helps in reducing the vibration, it is very much important to efficiently design the coefficients of stiffness and the damping coefficients of these mechanical components. Thus, number of washing machine vibration analysis models at various speeds have been proposed at various unbalanced condition in this paper. In this paper we are concentrating on reducing the vibrations of the drum with the help of maintaining exact coefficient of dampers and stiffness of the springs.
\end{abstract}

Keywords: Spring Stiffness, Damping Coefficient, Vibration Reduction

\section{INTRODUCTION}

Recent environmental awareness requires the improvement of washing efficiency. Talking about efficiency, the first we needs to concentrate on optimization. The basic factors that leads to optimization in a washing machine are its washing capacity, power consumption, and vibration. In terms of power consumption, major amount of all electrical energy generated is consumed in industry as HT line.the industrial drive system has been classified in two types. One group includes electrical components requiring speed control systems for different applications for example, machining tools and measuring machines for which precision in operation is required.

Washing machines has been developed to be automatic machines with several milestones. Automatic washing machine scan be characterized into two types according to loading types, namely, top loading model with vertical axis and front loading horizontal axis.

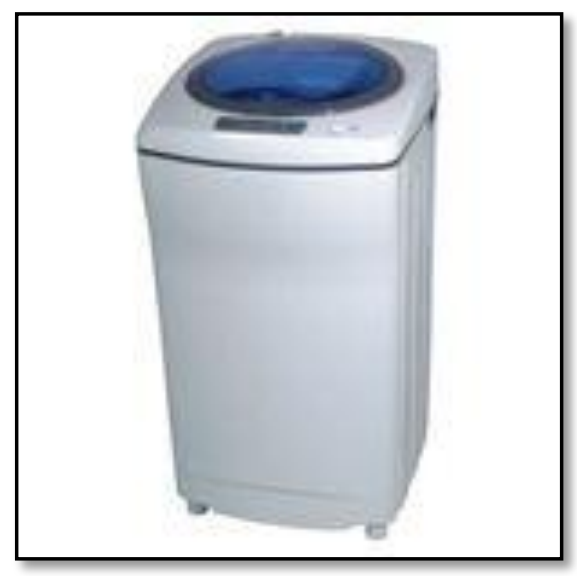

- ***.

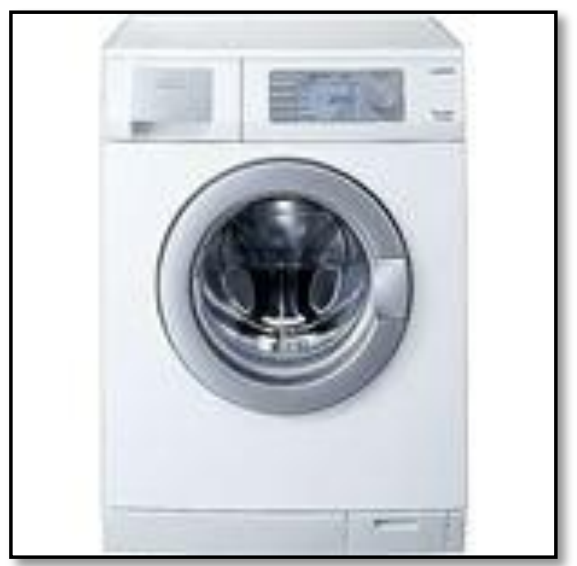

Figure: 1 Vertical and horizontal axis washing machine

Front loading type had become more common in Europe, and top loading models were popular in USA and Asia. Recently, front loading type has been increased in the United States and Asia.

In a fully automatic washing machine with variable speed control, an unbalanced mass of clothes against gravity in a spin drum can cause vibration problems during speed drying stage. This phenomenon mostly occurs most frequently during spin-drying stage, as speed of drum rotation is far more higher causing the clothes to be acts as a unbalanced masses and get pressed against the inner wall of the spin drum, excessive vibration can occur during the spin-drying stage. Same problem is more severe in horizontal axis washing machine as force of gravity causes addition of unbalanced mass. 
In a horizontal axis washing machine, vibration starts with outer tub causes vibration of the frame and then force transfers to the supports and then to the frame and to the floor. Thus it is vary much important to efficiently design the stiffness coefficients and the coefficient of damping of these mechanical components. Thus, different washing machine vibration analysis models have been proposed at various speeds in this model.

The purpose of this research is to generate a vibration analysis model of a front loaded washing machine washing machine that can analyze the vibration of the outer tub in various unbalanced conditions.. The characteristics of the spring damper and investigated experimentally at different speeds and compared with analysis results

\subsection{Problem Statement}

In washing machine vibration of washing machine generate vibration of outer frame and force transferred from frame to the floor. Although springs and dampers are already there but they are not providing enough vibration suspension based on variation in speed which may cause extra and minimum suspension than the requirements at higher and lower speeds.

Recently it is essential to reduce these vibrations

\section{MODELLING}

All the parts of the drum type washing machine were modeled using CATIA modeling software. Different parts of the washing machine were designed according to their original dimensions and constitution. These parts were then assembled and a working model was made.

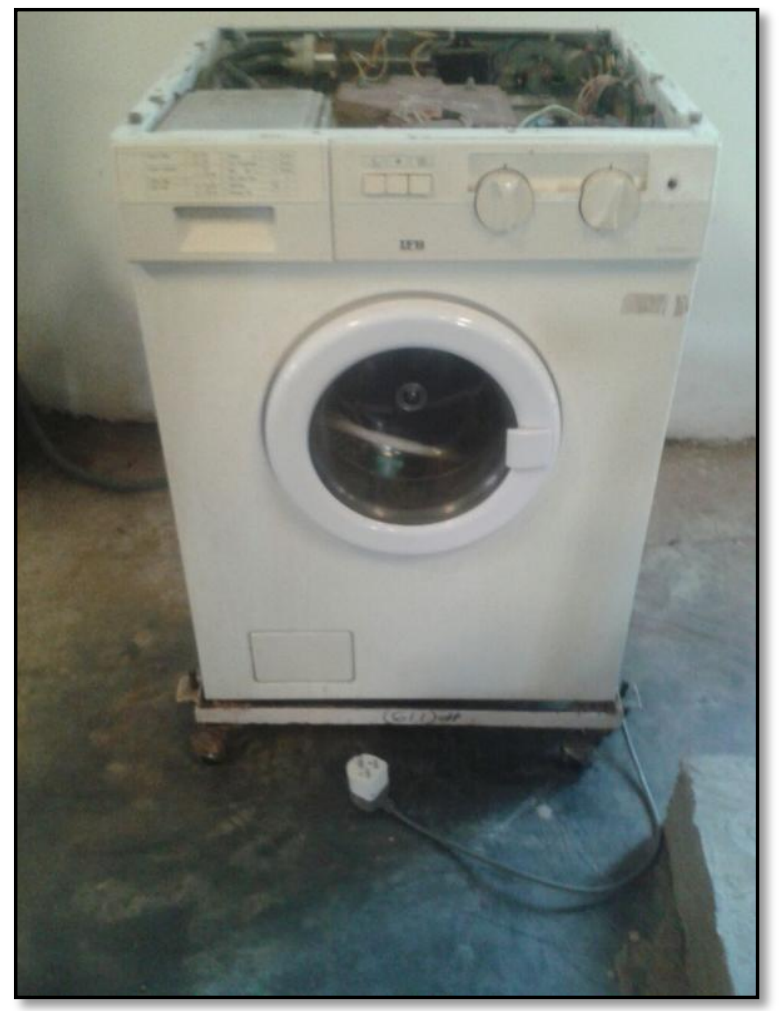

Figure: 2 Actual image of washing machine
All other parts like springs, rotatingdrum, main bady and cut sections are also drawn in catia.

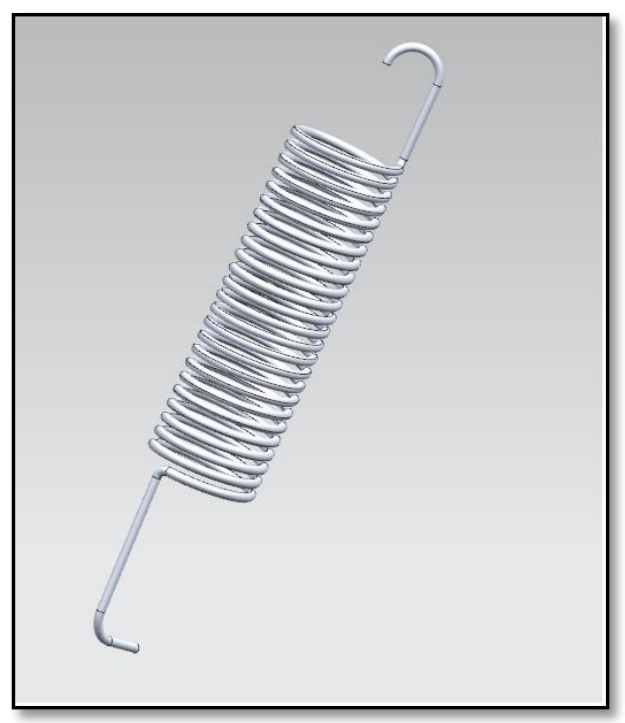

Figure -3: Spring

Different parts of the washing machine were designed according to their original dimensions and constitution. These parts were then assembled and a working model was made.

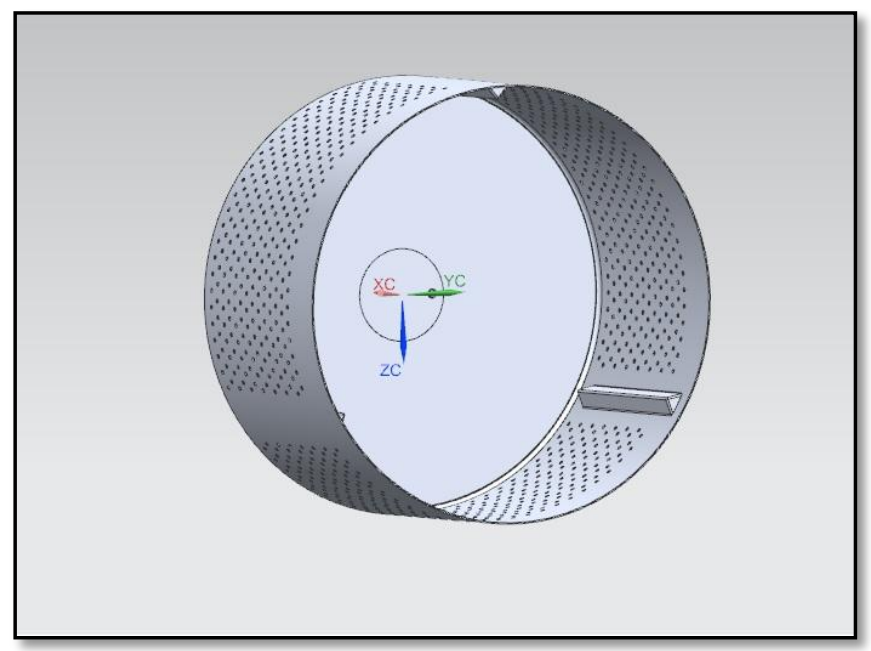

Figure: 4 Inner tub

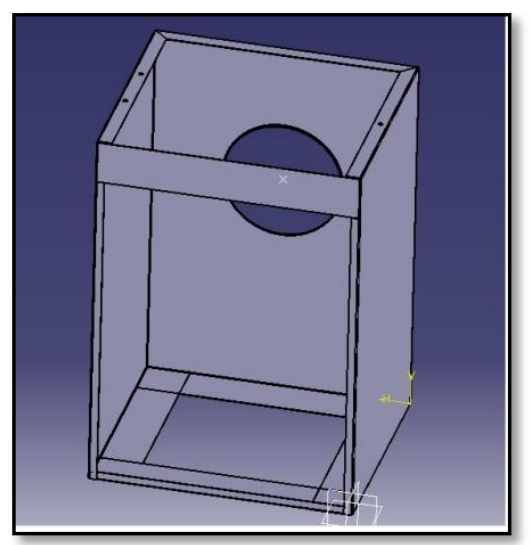

Figure:5 Frame Body 


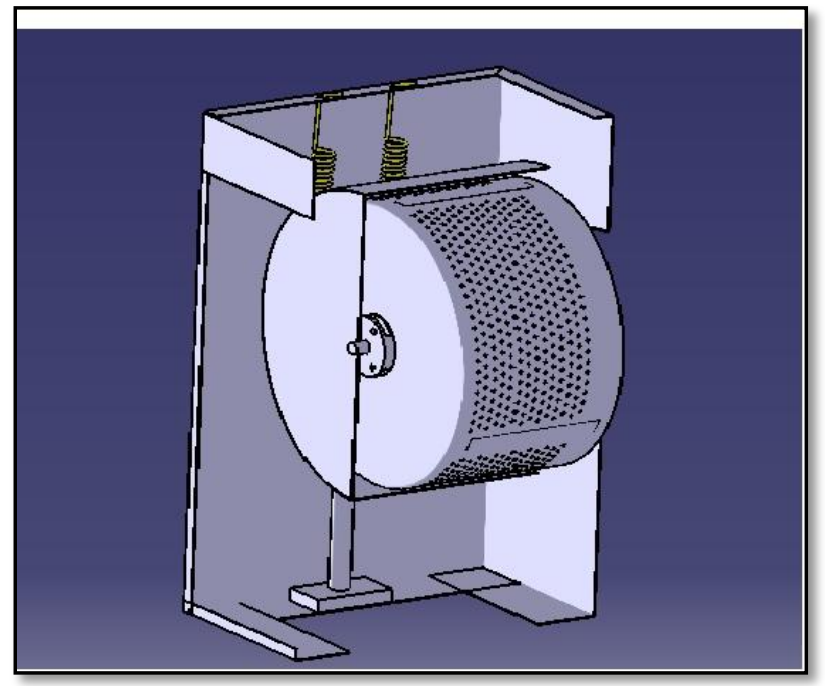

Figure: 6 Cut sections

\section{THEROTICAL ANALYSIS}

Theoretical analysis started by referring suitable data for model that has been used during experimentation. The calculations includes the values of displacement for the drum at various speeds using an unbalanced mass of $50 \mathrm{gm}$. One of the sample calculations are as follows,

\section{$\mathbf{c}=\mathbf{1 2 0} \mathrm{N}-\mathrm{s} / \mathbf{m}$}

$\mathrm{k}_{\mathrm{e}}=(5000+5000)+7500$

$k_{\mathrm{e}}=17500 \mathrm{~N} / \mathrm{m}$

$\mathrm{m}=15 \mathrm{~kg}$

$\omega_{n}=34.1565 \mathrm{rad} / \mathrm{sec}$

$$
\begin{gathered}
\omega_{n}=\sqrt{\frac{k_{e}}{m}} \\
\omega_{n}=\sqrt{\frac{17500}{15}}
\end{gathered}
$$

$$
\begin{gathered}
\xi=\frac{c}{c_{c}} \\
\xi=\frac{c}{2 m \omega_{n}} \\
\xi=\frac{120}{2 \times 15 \times 34.1565} \\
\xi=\mathbf{0 . 1 1 7 1}
\end{gathered}
$$

$F_{1}=\sqrt{F_{s 1}^{2}+F_{d 1}^{2}}$

$F_{1}=\sqrt{\mathbf{1 3 6 . 5}^{2}+68.6123^{2}}$

$F_{1}=152.774 \mathrm{~N}$

Above calculations are repeated for the further speeds for the calculations of displacement. Following table shows the calculations of different parameters at various speeds of washing machine which further requires for analysis.

\section{EXPERIMENTAL ANALYSIS}

The Washing machine was put under variable speed conditions in the Spin Drying stage, with the help of a Dimmer Stat. The Displacements were recorded with the help of FFT Analyser of drum and frame. According to the requirement of our project Single channel FFT analyser was used to measure the displacement caused by vibration Different readings were taken on different rpm.

Variable speed condition in washing machine is achieved by applying dimmerstat in main armature winding of washing machine. This arrangement provides us the feasibility to work in any range of speeds as per our requirement.
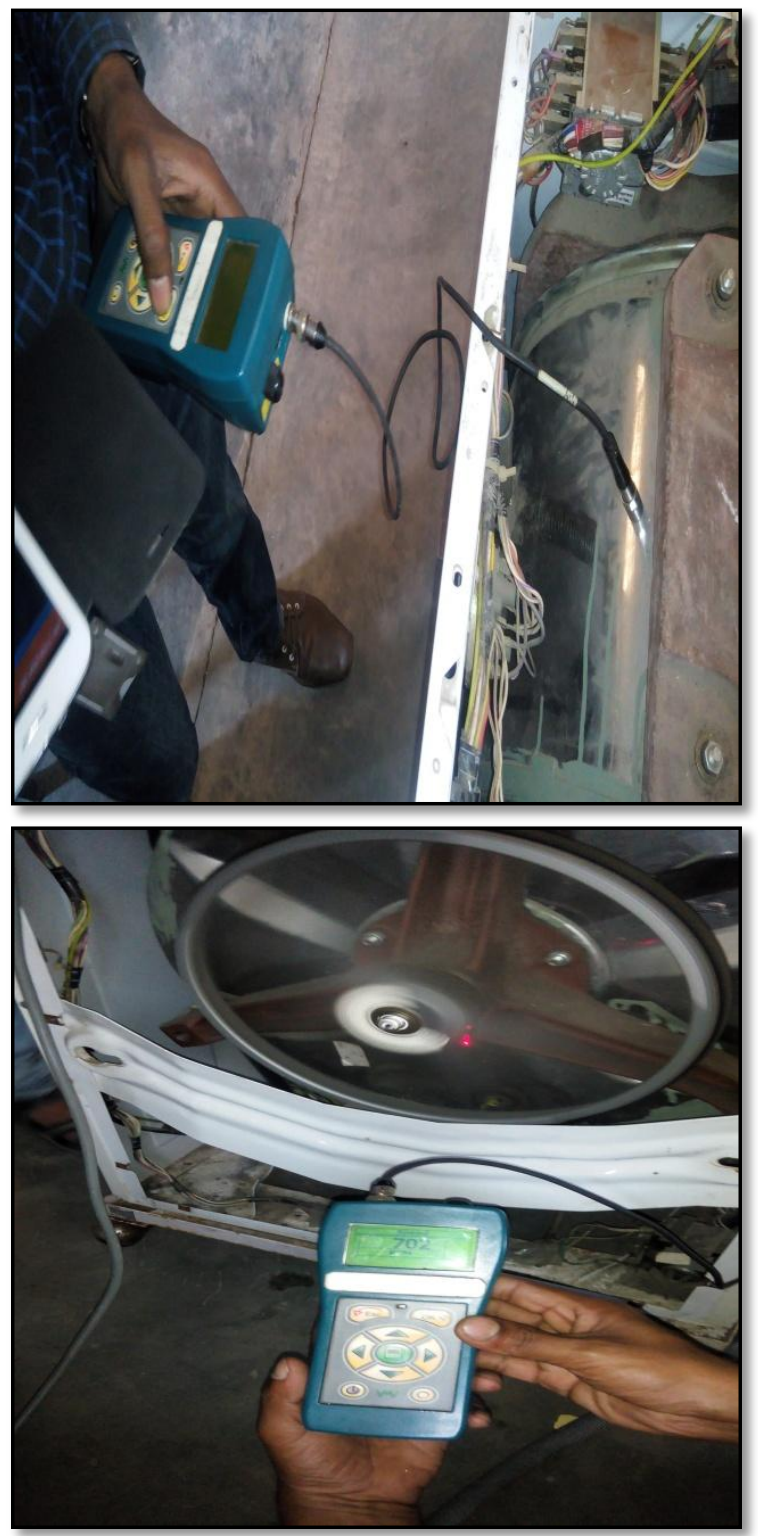

Figure: 7 displacement measurement using FFT

\section{SOFTWARE ANALYSIS}

Since the majority of industrial components are made of metal, most FEA calculations involve metallic components. The analysis of metal components can be carried out by either linear or nonlinear stress analysis. Which analysis approach you use depends upon how far you want to push the design.Analysis has been performed to verify the values of displacement at various speeds with experimental values of displacement at various speeds. 


\section{1 ] INPUT PARAMETERS -}

\section{i) FREQUENCY - 700 RPM}

ii) FORCE ON SHAFT - $152.774 \mathrm{~N}$

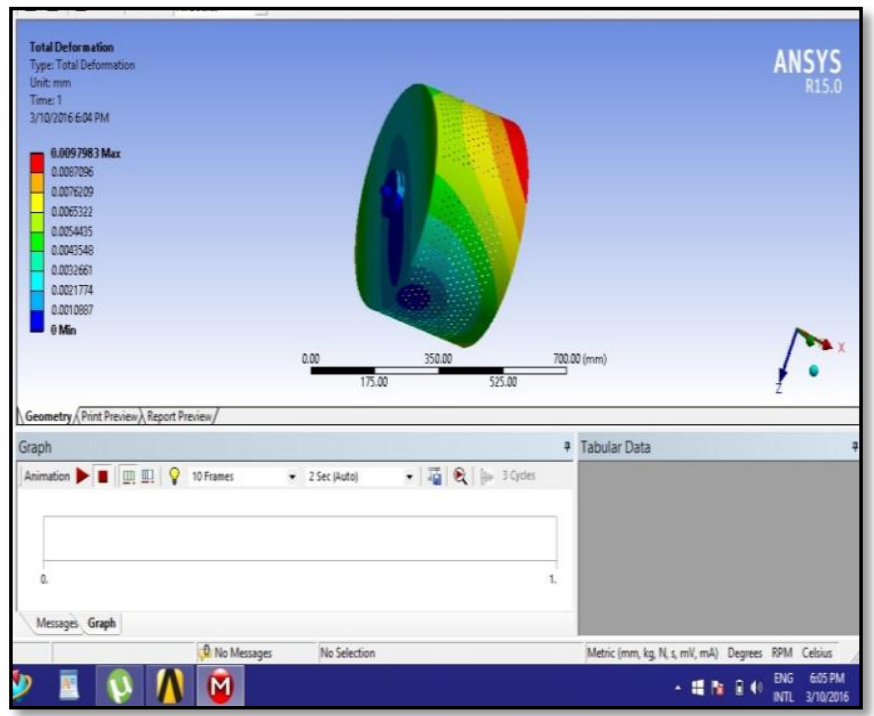

Figure: 8 Displacement measurement using software at 700RPM

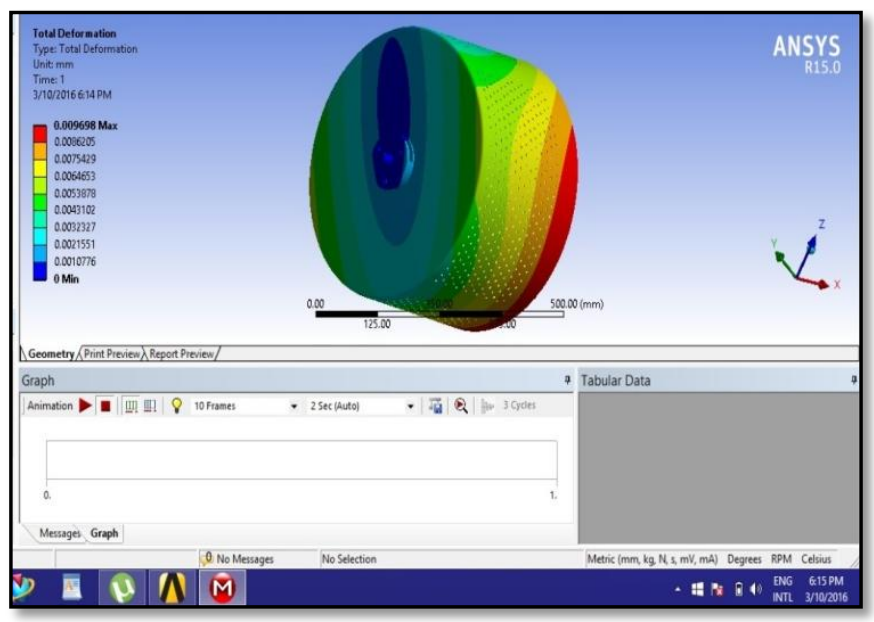

Figure:9 Displacement measurement using software at 725RPM

The same software results has achieved at different speeds for the verification of displacement. By comparing the experimental and software results we can easily identify the exact values of displacement at different speeds.

\section{CONCLUSION}

The results are shown in tabular form below. Theoretical analysis is based on ideal conditions and appropriate assumptions. Based on old model new criteria was made to for designing new model.

$\mathrm{N} 1=700 \mathrm{rpm}$, to find value of $\omega_{\mathrm{n}}$ let's take $\mathrm{X}=8$

$\omega=\frac{2 \pi \mathrm{N}_{1}}{60}$

$\omega=\frac{2 \times \pi \times 700}{60}$ $\omega=75.9218 \mathrm{rad} / \mathrm{sec}$

Assuming, $\frac{\omega}{\omega_{\mathrm{n}}}=\mathrm{z}$

$8=\frac{\left(\frac{\mathrm{m}_{0} \mathrm{e}}{\mathrm{m}}\right)(\mathrm{z})^{2}}{\sqrt{\left(1-\mathrm{z}^{2}\right)^{2}+(2 \xi(\mathrm{z}))^{2}}}$

$8=\frac{\left(\frac{0.5 \times 0.185}{15}\right)(\mathrm{z})^{2}}{\sqrt{\left(1-\mathrm{z}^{2}\right)^{2}+(2 \times 0.1171 \times(\mathrm{z}))^{2}}}$

$\mathrm{z}=2.05122$

Now,

$\frac{\omega}{\omega}=\mathrm{Z}$

$\frac{75.9218}{\omega_{\mathrm{n}}}=2.05122$

$\omega_{\mathrm{n}}=37.01299 \mathrm{rad} / \mathrm{s}$

Now to find $\mathrm{k}$,

$\omega_{\mathrm{n}}=\sqrt{\frac{\mathrm{k}}{\mathrm{m}}}$

$37.01299=\sqrt{\frac{\mathrm{k}}{15}}$

$\mathrm{k}=20552.76 \mathrm{~N} / \mathrm{m}$

Similarly we had done all the calculation for the different natural frequency \& spring stiffness at different amplitude value.

By varying the dimensions and controlling parameters axial and radial stiffness was calculated shown in table.From this tabular value we can calculate the different stiffness value of spring at the given value of the speed in rpm at different natural frequency \& different amplitude value by interpolating the value given below in the table.

\section{FUTURE SCOPE}

As optimization leads to formation of fault free system, in future we may also concentrate on damper variation to optimize vibration. In this paper we have concentrated on spring stiffness by keeping damping coefficient constant. In future we can vary damping coefficient by keeping spring stiffness constant.

\section{REFERENCES}

[1]. Y.Yokoi, Y.Sonoda, A.Okonogi, Y.Tomigashi, T Kawaguchi, Vibration Control System for the Drum TypeWasher/Dryer, SANYO TECHNICAL REVIEW, Vol. 35 No. 2, (2003), pp76-86

[2]. O.S.Turkay, I.T.Sumer, A.K.Tugcu, B.Kiray, Modeling and Experimental Assessment of SuspensionDynamics of a Horizontal-Axis Washing Machine, Journal of Vibration and Acoustics, vol. 120, (1998),pp534-543

[3]. E.Papadopoulos, I.Papadimitriou, Modeling, Design and Control of a Portable Washing Machine during theSpinning Cycle, IEEE/ASME International Conference on Advanced Intelligent Mechatronics Proceedings,Vol. 2001 No. Vol. 2, (2001), pp899-904 